\title{
CIRURGIA AMBULATORIAL: DO CONCEITO À ORGANIZAÇÃO DE SERVIÇOS E SEUS RESULTADOS
}

\author{
OUTPATIENT SURGERY: THE CONCEPT, ORGANIZATION OF THE PROGRAM AND ITS` RESULTS
}

José Sebastião dos Santos ${ }^{1}$, Ajith Kumar Sankarankutty ${ }^{1}$, Wilson Salgado $\mathrm{Jr}^{1}$, Rafael kemp ${ }^{2}$, Elias Paim Leonel ${ }^{3}$, Orlando de Castro e Silva $\mathrm{Jr}^{1}$

\begin{abstract}
1'Docentes, ${ }^{2}$ Médico Assistente e Pós Graduando. Divisão de Cirurgia Digestiva do Departamento de Cirurgia e Anatomia da FMRP-USP. ${ }^{3}$ Médico Residente da Disciplina de Ortopedia e Traumatologia do Hospital das Clínicas da FMRP-USP.

Correspondência: José Sebastião dos Santos. Divisão de Cirurgia Digestiva do Departamento de Cirurgia e Anatomia da FMRP-USP. Av. Bandeirantes, 3900, 14049-900 - Ribeirão Preto / SP. (email: jsdsanto @fmrp.usp.br)
\end{abstract}

Santos JS, Sankarankutty AK, Salgado Jr W, Kemp R, Leonel EP, Castro e Silva Jr O. Cirurgia ambulatorial: Do conceito à organização de serviços e seus resultados. Medicina (Ribeirão Preto) 2008; 41 (3): 274-86.

RESUMO: O desenvolvimento da cirurgia videoendoscópica, a introdução de drogas anestésicas de duração curta com efeitos colaterais reduzidos e as necessidades didáticas e assistenciais associadas à imperativa gestão racional dos leitos hospitalares têm contribuído para a expansão dos limites da cirurgia ambulatorial, em nosso meio. Esse trabalho traz os fundamentos da cirurgia ambulatorial nos seus aspectos conceituais, técnicos, éticos e legais. Nesse contexto, o processo de implantação de serviços de cirurgia ambulatorial de pequeno e grande porte e a evolução dos seus resultados são descritos. Finalmente, destaca-se o papel desse movimento técnico e político em sintonia com as diretrizes do Sistema Único de Saúde para a consolidação de uma rede de serviços de cirurgia ambulatorial que integra o Centro de Saúde Escola, o Hospital Estadual e o Hospital das Clínicas e conta com a cooperação da Faculdade de Medicina de Ribeirão Preto da Universidade de São Paulo.

Descritores: Cirurgia Ambulatorial. Videolaparoscopia. Educação Médica. Colecistectomia. Sistema Único de Saúde.

\section{1- INTRODUÇÃO}

A mudança do pensamento médico sobre o valor terapêutico do repouso no pós-operatório abriu perspectiva para o desenvolvimento da cirurgia ambulatorial. Entre 1800 e 1940, o repouso era visto como a maior contribuição para a recuperação pós-operatória. Todavia, a partir das experiências assistenciais vivenciadas na $2^{\mathrm{a}}$ Grande Guerra Mundial aumentaram as observações apoiando a redução, não só do repouso pós-operatório, mas também do período de internação ${ }^{1}$.

Por outro lado, mesmo antes deste período, há registros de experiências bem sucedidas com as práticas da cirurgia ambulatorial na América do Norte e na Europa. Em 1909, Nicholl, do Royal Glasglow Hospital for Children, apresentou os resultados do tratamento cirúrgico ambulatorial de quase 9.000 crianças, salientando que eram tão satisfatórios quanto àqueles obtidos com as operações realizadas em regime hospitalar $^{2}$.

No Canadá, em meados de 1940, no Shouldice Hospital, foi iniciado um programa para realização de hernioplastias inguinais para adultos, sob anestesia local, com curta permanência hospitalar ${ }^{3}$.

A era moderna da cirurgia ambulatorial começou na década de 60, do século passado, no Butterworth Hospital, em Michigan, Estados Unidos da América (EUA $)^{4}$. O tratamento de pacientes sem internação passa a contar com apoio da anestesiologia. A cirur- 
gia ambulatorial torna-se uma prática segura, se empreendida com a seriedade e controles rigorosos por parte do cirurgião e do anestesiologista, não havendo razões para a ocorrência de maior incidência de complicações em comparação ao tratamento feito em ambiente hospitalar ${ }^{5}$.

No Brasil, as experiências registradas representam iniciativas isoladas de alguns entusiastas ou de algumas instituições ${ }^{6-9}$, que às vezes se desenvolvem por necessidade de natureza didática e assistencial $^{10}$ ou de redução das listas de espera para cirurgia $^{11}$, sem contar com o apoio formal de políticas públicas de educação e saúde.

Nesse contexto, houve um crescente aperfeiçoamento dos conceitos e das práticas da cirurgia ambulatorial, sobretudo em alguns países da Europa e nos EUA. O desenvolvimento das técnicas anestésicas e cirúrgicas, a incorporação de recursos para prevenção da dor, da náusea e do vômito, a melhoria da qualidade dos estabelecimentos e do sistema de saúde, além da redução dos custos da internação têm contribuído para o desenvolvimento da cirurgia ambulatorial $^{12}$ (Figura 1).
Em função do porte da operação, das características do serviço e das condições dos pacientes, as unidades de cirurgia ambulatorial podem funcionar com três modelos ${ }^{13}$ :

- Dispensação após o procedimento;

- Permanência por um curto tempo para recuperação;

- Pernoite.

Em função do porte da operação e dos cuidados pós-operatórios, a cirurgia ambulatorial pode ser classificada como de pequeno ou de grande porte

- " A cirurgia ambulatorial de pequeno porte é realizada geralmente sob anestesia local com alta imediata do paciente: incluem as operações feitas no consultório ou ambulatório (retirada de lesões tumorais da pele e do subcutâneo, postectomia, vasectomia, hemorroidectomia, polipectomias endoscópicas em casos selecionados);

- A cirurgia ambulatorial de grande porte é realizada sob qualquer modalidade de anestesia, sendo necessário um período de monitorização ou recuperação pós-operatória (herniorrafias inguinais, safenectomias, papilotomia endoscópica, colecistectomia e hernioplastias hiatais por videolaparoscopia).

\section{3- TIPOS DE UNIDADES DE CIRUR- GIA AMBULATORIAL}

A organização das unidades de cirurgia ambulatorial pressupõe a mesma qualidade e segurança das unidades de internação. No Estado de São Paulo, os estabelecimentos para realização de procedimentos médicos cirúrgicos ambulatoriais são classificados em três tipos, segundo a Resolução da Secretaria de Estado da Saúde ${ }^{14}$ :

\section{1- Unidade ambulatorial tipo I}

Consultório médico que mediante adaptação possibilita a realização de proce-

\section{2- CIRURGIA AMBULATORIAL}

\section{1- Aspectos conceituais}

As operações em regime ambulatorial são realizadas sob qualquer tipo de anestesia, não demandam internação hospitalar e a permanência do paciente no serviço não deve exceder 24 horas. dimentos médico-cirúrgicos de pequeno porte, sob anestesia local.

\section{2- Unidade ambulatorial tipo II}

Ambulatórios isolados, centros de saúde e unidades básicas de saúde onde é possível a realização de procedimentos de porte médio, com anestesia local ou loco - regional (com ou sem sedação). Além das 
salas com as devidas especificações, a Unidade deve contar com sala de recuperação ou de observação do paciente.

\section{3- Unidade ambulatorial do tipo III}

O estabelecimento de saúde que, anexo ou não a um hospital geral ou especializado, possibilita a realização de procedimentos em regime ambulatorial ou de internação, em salas cirúrgicas próprias ou do centro cirúrgico do hospital, mediante apoio da sua infraestrutura (Serviço de Nutrição e Dietética, Centro de Esterilização de Material e Lavanderia, Central de Gases, Central de Vácuo, Central de Ar Comprimido, Central de Ar Condicionado, Sistema de Coleta de Lixo, dentre outros) .

\section{4- REGULAMENTAÇÃO DA PRÁtiCA DA CIRURGIA AMBULATORIAL}

A realização das cirurgias ambulatoriais é regulamentada pela portarias do Conselho Federal de Medicina ${ }^{15,16}$. Os atos cirúrgicos e ou endoscópicos, em regime ambulatorial, devem levar em conta as seguintes condições:

\section{1- Condições da Unidade}

a) Condições estruturais e sanitárias do ambiente com estrutura para esterilização e desinfecção dos instrumentos de acordo com as normas vigentes;

b) Registro de todos os procedimentos realizados;

c) Condições mínimas para a prática da anestesia; ${ }^{16}$

d) Garantia de internação, caso haja necessidade;

e) Garantia de assistência pós-alta durante 24 horas por dia na unidade ou no sistema de saúde.

\section{2- Critérios de seleção do paciente}

a) Pacientes hígidos ou com distúrbio sistêmico moderado decorrente de doenças crônicas;

b) Procedimentos cirúrgicos que não necessitam cuidados especiais no pós-operatório;

c) Garantia de acompanhante adulto, lúcido e previamente identificado.

\section{3- Condições de alta do paciente}

a) Orientação no tempo e no espaço;

b) Estabilidade dos sinais vitais há pelo menos $60 \mathrm{mi}-$ nutos;

c) Ausência de náusea e vômitos;

d) Ausência de dificuldade respiratória;

e) Capacidade de ingerir líquidos; f) Capacidade de locomoção, se não houver contraindicação;

g) Sangramento mínimo ou ausente;

h) Ausência de dor de grande intensidade;

i) Ausência de retenção urinária;

j) Conhecimento por parte do paciente e do acompanhante, verbalmente e por escrito, da relação dos cuidados pós-anestésicos e pós-operatórios, bem como a determinação da Unidade para atendimento de eventuais intercorrências.

A decisão final de inclusão de pacientes para tratamento cirúrgico em regime ambulatorial envolve saberes multiprofissionais de médicos cirurgiões e anestesiologistas, enfermeiros e assistentes sociais.

O cirurgião com base na anamnese e no exame físico indica a operação e faz a programação da sua realização mediante anuência do paciente, levando em conta aspectos fisiológicos, psicológicos e sociais, além, evidentemente, da avaliação anestésica. A maioria dos pacientes incluídos no programa de cirurgia ambulatorial deve pertencer à classe I e II do escore da Sociedade Americana de Anestesiologistas (ASA). Os pacientes classificados como ASA I não apresentam alterações orgânicas, fisiológicas, bioquímicas ou psiquiátricas enquanto os pacientes avaliados como ASA II apresentam alterações de pequena monta facilmente corrigíveis. Os pacientes classificados como ASA III devem ser operados em regime de internação por apresentar alterações sistêmicas graves ou, eventualmente, se reunirem condições de ser tratados em regime ambulatorial, o procedimento deve ser feito em unidades integradas a um hospital ${ }^{11}$.

O tratamento cirúrgico em regime ambulatorial conta com a cooperação do paciente e do acompanhante no pré e pós-operatório. Assim, faz-se necessário avaliar o estado psicológico dos candidatos. Adultos e crianças com distúrbios mentais, de atitude e de comportamento devem, de preferência, ser submetidos a tratamento cirúrgico, em regime de internação hospitalar.

Além das avaliações fisiológica e psicológica, uma avaliação dos suportes familiar e social do candidato à cirurgia ambulatorial é essencial. Não é recomendável que o paciente viaje por mais de uma hora após o procedimento, principalmente, se for por meio de transporte social. A dificuldade de acesso aos serviços de saúde para avaliação de complicações eventuais também limita a indicação. $\mathrm{O}$ apoio familiar é fundamental, considerando que parte do pré e do pós- 
operatório é transferido do hospital para a casa. Assim, a facilidade de comunicação e transporte, bem como de acessibilidade à assistência para avaliação de alguma intercorrência pós-operatória é de extrema relevância e precisa estar assegurada ${ }^{11}$.

A avaliação clínica sistematizada do paciente e da sua doença por meio de consensos pode revelar a possibilidade de realização do tratamento em regime ambulatorial e orientar a solicitação dos testes préoperatórios. Os testes recomendados para o ato anestésico estão no Quadro I. Não é admissível que os pacientes da cirurgia ambulatorial sejam submetidos a maior número de exames por não estarem hospitalizados ou em função do potencial cancelamento da operação ${ }^{17}$.

\section{Quadro 1- Principais testes pré-operatórios reco- mendados para o ato anestésico.}

IDADE < 50 ANOS, PACIENTE SAUDÁVEL - NENHUM TESTE.

\section{Eletrocardiograma}

- Paciente com idade maior ou igual a 50 anos

- Antecedente de hipertensão arterial

- História de doença cardiovascular

- Procedimento cardiotorácico

Rx de tórax

- Procedimento cardiotorácico

Testes bioquímicos no sangue

- Doença renal

- Doença metabólica (diabetes, adrenal, tiróide)

- Uso de diurético e quimioterápico

Exame de urina

- Procedimentos geniturinários

Hemograma

- Distúrbios hematológicos

A sistematização da solicitação de testes préoperatórios reduz em media $55 \%$ do total de exames com variação de $89 \%$ para o exame de urina e $17 \%$ para o hemograma ${ }^{18}$.

\section{5- CIRURGIA AMBULATORIAL - VANTA- GENS E DESVANTAGENS}

\section{1- Vantagens para os pacientes}

a) Alteração mínima na rotina do paciente e da família ${ }^{7}$.

b) Individualização do cuidado. A avaliação da relação médico-paciente tem o melhor desempenho em comparação com as outras áreas cirúrgicas ${ }^{19}$. c) Redução do risco de infecção hospitalar. A menor exposição ao ambiente hospitalar contribui para a redução das taxas de infecção hospitalar. As taxas de infecções globais em cirurgia, no ambiente hospitalar variam de 2 a $15 \%$ e recomenda-se que a taxa de infecção de ferida em cirurgia limpa deve ficar abaixo de $3 \%{ }^{20}$. Em cirurgia ambulatorial, os índices variam de zero ${ }^{21}$ a $4,4 \%{ }^{9}$.

d) Redução da incapacidade física com retorno mais rápido ao trabalho ${ }^{4}$.

e) Morbidade e mortalidade menores. A mortalidade é próxima de zero. As intercorrências mais freqüentes são as dores, as náuseas e os vômitos.

\section{2- Vantagens para o Sistema de Saúde}

a) Redução dos custos. A redução dos custos dos procedimentos realizados em regime de cirurgia ambulatorial quando comparada com o tratamento em regime de internação hospitalar é de 55\% nas unidades autônomas, $46 \%$ nas unidades satélites e $11 \%$ nas unidades integradas ${ }^{19}$.

b) Maior disponibilidade de leitos hospitalares. A introdução prática da cirurgia ambulatorial aumenta a disponibilidade de leitos hospitalares para a internação de pacientes com afecções cirúrgicas mais complexas $^{11}$.

\section{3- Desvantagens da cirurgia em regime ambu- latorial}

a) Não realização dos cuidados pré-operatórios: a inobservância do tempo de jejum e da realização dos exames específicos e de rotina pode contribuir para o adiamento ou a suspensão da cirurgia ${ }^{4}$.

b) Falta de transporte. Os familiares, os vizinhos ou o gestor de saúde devem ser mobilizados com antecedência para garantir o transporte $e^{4,11}$.

c) Falta de ajuda no domicílio. Os pacientes que moram sozinhos e não dispõem de familiares e amigos para a ajuda no domicílio para os cuidados gerais e específicos, e, principalmente, para garantir o retorno pós-operatório devem ser tratados em regime de internação ${ }^{4}$.

d) Suspensão da cirurgia em regime ambulatorial em detrimento de urgência, sobretudo nas unidades integradas ao hospital ${ }^{4}$.

e) Necessidade de permanência hospitalar além do esperado. A variabilidade das taxas de internação depende do tipo da afecção tratada, das características biológicas, psicológicas e sociais do paciente selecionado, da experiência do serviço e das condições sociais e econômicas da população de abrangência. 


\section{6- CIRURGIA AMBULATORIAL - CUIDADOS PERI-OPERATÓRIOS}

O sucesso da cirurgia ambulatorial depende de integração da equipe multiprofissional, de protocolos rigorosos de seleção e também da sistematização de condutas. As estratégias multimodais incluindo medidas pré-operatórias (controle de comorbidades, seleção, interrupção do tabagismo, cuidados nutricionais), trans-operatórias (antibioticoprofilaxia, técnica cirúrgica adequada e de preferência minimamente invasiva, técnica anestésica atualizada) e pós-operatórias (controle da dor e das náuseas, nutrição precoce) asseguram a efetividade da cirurgia ambulatorial ${ }^{22}$.

\section{1- Cuidados pré-operatórios}

a) Antibióticoprofilaxia: tem como objetivo prevenir infecções por agentes conhecidos ou suspeitos em pacientes de risco. Os princípios básicos são: 1) empregar em cirurgias que comprovadamente tenham sua taxa de infecção diminuída pelo uso do antibiótico; 2) utilizar agentes de primeira linha com ação efetiva contra germes específicos para cada tipo de cirurgia; 3) os níveis séricos do antibiótico devem ser máximos no momento da exposição do organismo ao agente infectante; 4) o emprego deve ser limitado à duração da cirurgia, ou no máximo 24 horas após.

b) Profilaxia da trombo-embolia pulmonar (TEP): a alta mortalidade e incidência, muitas vezes subestimada desta doença, cada vez mais têm sido objeto de atenção de profissionais da saúde. O tempo médio de profilaxia para pacientes cirúrgicos é de 7 a 10 dias. A maioria dos pacientes que são submetidos à cirurgia em regime ambulatorial não necessita de profilaxia farmacológica para TEP, pois as operações são de pequeno ou médio porte, o risco, em geral é baixo e a deambulação é precoce. Dessa forma, a profilaxia do TEP deve ser orientada de acordo com o risco calculado para cada paciente: ${ }^{23}$

Dentre as orientações, as que são fortemente recomendadas:

- Utilizar apenas métodos mecânicos quando há risco elevado de sangramento;

- Baixo risco: procedimento cirúrgico de porte pequeno, idade menor que 40 anos e sem fato- res de risco adicionais empregar apenas o método mecânico;

- Risco Moderado: procedimento cirúrgico de porte pequeno e idade entre 40-60 anos ou com algum fator de risco, empregar heparina 5000 unidades no subcutâneo 12/12h ou heparina de baixo peso molecular $(\mathrm{HBPM}) \leq 3400 \mathrm{U}(20 \mathrm{mg}$ enoxaparina)

- Alto Risco: cirurgias menores e idade maior que 60 anos; cirurgias maiores se idade entre 40-60 anos ou com fatores de risco, utilizar heparina 5000 unidades no subcutâneo 8/8h ou HBPM > 3400 U (40mg enoxaparina)

- Altíssimo risco: múltiplos fatores de risco (> 40 anos, câncer, TEP prévia), artroplastia de quadril ou joelho, trauma maior, lesão de medula, utilizar medicação do alto risco mais medidas mecânicas.

- Cirurgia laparoscópica sem fatores de risco: orienta-se apenas a mobilização precoce

- Cirurgia laparoscópica com fatores de risco: recomenda-se o emprego de um ou mais destes métodos: heparina, HBPM e/ou mecânico.

A profilaxia medicamentosa, no risco moderado, é recomendada duas horas antes da cirurgia (anestesia geral) ou 12 horas antes da punção em bloqueio espinhal. Já no caso de alto risco deve ser realizada 12 horas antes tanto da anestesia geral, quanto da punção do bloqueio espinhal.

c) Orientações aos pacientes: Conforme frisado anteriormente, uma das desvantagens da cirurgia ambulatorial é a possibilidade do paciente não seguir as orientações fornecidas. Para tanto, recomenda-se, além de orientar verbalmente o paciente e seus acompanhantes, fornecer um Manual com os cuidados básicos:

- Jejum de 4 horas se anestesia local ou de 8 horas se outra anestesia;

- Não realizar tricotomia no domicílio, apenas tomar banho antes;

- Vir acompanhado de um adulto que se responsabilize pelo retorno do paciente para casa;

- Suspender tabagismo pelo menos 30 dias antes.

d) Compensação de co-morbidades: Adiar a cirurgia até que todas as comorbidades infecciosas e não infecciosas estejam compensadas. 


\section{2- Cuidados pós-operatórios}

a) Re-introdução da dieta: a re-alimentação deve ser a mais precoce possível, desde que o paciente esteja bem acordado. Os cuidados com as náuseas e vômitos merecem atenção especial. ${ }^{24}$

b) Dor: sintoma muito freqüente no pós operatório e que precisa ser inibido e tratado com rigor. A dor produz diversos efeitos deletérios, tais quais: problemas psicológicos, imunossupressão, diminuição da perfusão tissular, aumento do consumo de oxigênio, alteração respiratória, aumento do catabolismo e diminuição da movimentação com riscos de trombose venosa. Reforça-se a opção por terapias multimodais, que envolvem mais de um princípio analgésico, com medicamentosos anti-inflamatórios não esteroidais, a dipirona, o acetaminofeno e os opióides.

c) Náuseas e vômitos: são responsáveis por aumentar a morbidade cirúrgica e prolongar a internação. Os fatores de risco são:

- Fatores individuais: gênero feminino, história prévia de náusea e vômito pós-operatório, cinetose e ansiedade;

- Fatores cirúrgicos: procedimento de longa duração, cirurgias abdominais, laparoscopia e cirurgia otorrinolaringológica;

- Fatores anestésicos: agentes inalatórios, óxido nitroso, etomidato, opióides, neostigmine.

Segundo Consenso publicado em $2003^{25}$, no risco baixo não é necessária a realização de profilaxia. No risco moderado recomenda-se monoterapia, e, eventualmente, com associação medicamentosa. Já no risco elevado, sugere-se o uso de dois ou três medicamentos. Dentre eles pode ser empregado os bloqueadores serotoninérgicos (ondansetrona) no final da cirurgia, a dexametasona antes da indução anestésica e/ou o droperidol, dentre outros.

Ainda, recomenda-se:

- Uso de propofol no intuito de reduzir a náusea das primeiras 6 horas;

- Anestesia loco-regional, que tem menor incidência de náusea;

- Doses menores de neostigmine na reversão anestésica;

- Fluidoterapia adequada para evitar hipotensão;

- Fornecimento elevado de oxigênio durante a cirurgia;

- Diminuição do uso de opióides e analgesia adequada. d) Cuidado com curativo e pontos: após 24 a 48 horas, a incisão está vedada em virtude da epitelização e neste momento o curativo pode ser removido. A troca de curativo, caso seja necessária antes deste período, deve ser realizada de forma asséptica.

O momento da retirada de pontos depende dos seguintes fatores: fio utilizado, modalidade de ponto, tipo e extensão da incisão, se foram respeitadas as linhas de força da pele e as condições locais e sistêmicas. De forma geral, recomenda-se retirar os pontos no sétimo dia pós-operatório.

\section{7- PRINCIPAIS PROCEDIMENTOS CIRÚR- GICOS REALIZADOS EM REGIME AM- BULATORIAL}

\begin{tabular}{|c|c|}
\hline Especialidade & Procedimento \\
\hline Cirurgia Geral & $\begin{array}{l}\text { Biópsia e exérese de lesões de pele, } \\
\text { subcutâneo, anexos e partes moles, } \\
\text { laparoscopia diagnóstica, colecistec- } \\
\text { tomia por minilaparotomia e videola- } \\
\text { paroscopia, varicectomia, herniorra- } \\
\text { fia de parede abdominal, hemorroi- } \\
\text { dectomia, hernioplastia hiatal por ví- } \\
\text { deolaparoscopia }\end{array}$ \\
\hline
\end{tabular}

Cirurgia pediátrica Hérnia de parede abdominal, postectomia

Dermatologia Biópsia e exérese de lesões cutâneas

Endoscopia Endoscopia digestiva alta e baixa para diagnóstico e terapêutica (polipectomia, papilotomia, esclerose de varizes)

Ginecologia Curetagem uterina, cirurgia da vulva, retirada de nódulos mamários, laparoscopia diagnóstica e terapêutica (laqueadura)

Oftalmologia Catarata, cirurgia do estrabismo

Otorrinolaringologia Amidalectomia, adenoidectomia, miringotomia laringoscopia, polipectomia, septoplastia, timpanoplastia

Ortopedia

Artroscopia, liberação do túnel do carpo, exérese de cisto sinovial.

Urologia Cistoscopia, orquipexia, vasectomia, postectomia, hidrocelectomia, varicocelectomia 


\section{8- AVALIAÇÃO DA ORGANIZAÇÃO E DOS RESULTADOS DOS SERVIÇOS DE CIRUR- GIA AMBULATORIAL IMPLEMENTADOS PELO COMPLEXO HOSPITAL DAS CLÍ- NICAS E CENTRO DE SAÚDE-ESCOLA DA FMRP-USP}

\section{1- Cirurgia ambulatorial no Centro de Saúde Escola}

A revisão do currículo médico da Faculdade de Medicina de Ribeirão Preto da Universidade de São Paulo (FMRP-USP) implementada, a partir de 1993, e a organização do acesso aos serviços assistenciais dos SUS, induzida pelo HCFMRPUSP, a partir de 1996 e fortalecida no ano de 2000, por meio da adoção da sistemática de referências e contra-referências e da regulação médica das urgências ${ }^{26,27}$, motivaram a estruturação de serviços com finalidades didáticoassistenciais voltados à prática da pequena e da grande cirurgia ambulatorial.

Com o objetivo de desenvolver as práticas cirúrgicas na atenção básica e no programa de saúde da família voltadas para as atividades da graduação, foi organizado, no ano de 1997, no Centro de Saúde Escola (CSE) da FMRP-USP, o Serviço de Cirurgia Ambulatorial para cirurgias de pequeno porte para tratamento de lesões da pele, subcutâneo e anexos, sob anestesia local (Unidade Tipo I) ${ }^{10}$. No ano de 2005 foram inauguradas as novas instalações para a cirurgia ambulatorial no CSE-FMRP-USP com características de Unidade do Tipo II.

O serviço foi organizado no seu eixo assistencial para atender às necessidades da população do Distrito Oeste da cidade de Ribeirão Preto. Por outro lado, na vertente didática, deve atender às necessidades de formação do graduando de medicina do $5^{\circ}$ e $6^{\circ}$ anos e os médicos do programa de residência em cirurgia geral e saúde de família, os quais realizam os procedimentos e vivenciam sob supervisão direta de médicos e docentes da cirurgia as etapas da assistência em cirurgia ambulatorial:- avaliação do doente e da doença, a indicação do procedimento, a seleção e o tratamento. Esse processo permite o desenvolvimento do conceito de cirurgia ambulatorial e o aprendizado das bases técnicas da cirurgia e dos cuidados préoperatórios.

De 1997 a 2002 foram tratados, neste serviço, 1806 pacientes; sendo que 1434 pacientes foram classificados como ASA I, 327 como ASA II e 45 como ASA III, de acordo com a American Society of Anesthesiologists (Tabela I). No período foram tratadas 1203 lesões tumorais, das quais $91(3,88 \%)$ de natureza maligna (Tabela II).
Tabela I: Características dos pacientes avaliados (1997-2002).

\begin{tabular}{lcccccc}
\hline & Pacientes & \multicolumn{3}{c}{ Sexo } & ASA \\
Ano & Avaliados & Masculino & Feminino & I & II & III \\
\hline 1997 & 491 & 258 & 233 & 437 & 52 & 2 \\
1998 & 398 & 187 & 211 & 311 & 84 & 3 \\
1999 & 227 & 74 & 153 & 173 & 40 & 14 \\
2000 & 121 & 44 & 77 & 86 & 34 & 1 \\
2001 & 233 & 97 & 136 & 167 & 61 & 5 \\
2002 & 336 & 128 & 208 & 260 & 56 & 20 \\
\hline Total & 1806 & 788 & 1018 & 1434 & 327 & 45 \\
\hline
\end{tabular}

Tabela II: Natureza das lesões tratadas (19972002).

\begin{tabular}{lrr}
\hline Lesão & \multicolumn{1}{c}{$\mathrm{N}^{\mathrm{o}}$} & \multicolumn{1}{c}{$\%$} \\
\hline Trauma superficial & 214 & 9,12 \\
Cisto & 344 & 14,66 \\
Unha encravada & 102 & 4,34 \\
Outras lesões não tumorais & 312 & 13,29 \\
Tumor benigno & 1203 & 51,26 \\
Tumor pré-maligno & 81 & 3,45 \\
Tumor maligno & 91 & 3,88 \\
\hline Total & 2347 & 100,00 \\
\hline
\end{tabular}

\section{2- Colecistectomia laparoscópica em regime ambulatorial}

As vantagens da cirurgia por videolaparoscopia e os progressos da anestesiologia para anestesia geral 
em procedimentos ambulatoriais ${ }^{28}$, associados ao preparo educacional dos pacientes e dos profissionais de saúde, têm possibilitado o tratamento dos pacientes com colelitíase sintomática em regime de cirurgia ambulatorial. Desta forma, associando os progressos da cirurgia videolaparoscópica e a estratégia dos mutirões da saúde, a colecistectomia videolaparoscópica passou a ser realizada em regime ambulatorial ${ }^{11}$.

A colecistectomia laparoscópica em regime ambulatorial é segura e efetiva em pacientes selecionados que desejam a operação, residem próximo ao hospital e dispõem de acompanhante responsável pelos cuidados após a alta. Aqueles pacientes que sofreram cirurgia abdominal alta prévia ou que apresentam suspeita de coledocolitíase ou colecistite aguda são geralmente excluídos do tratamento em regime ambulatorial ${ }^{29}$.

Em meta-análise de estudos que incluíram 215 pacientes no grupo de colecistectomia ambulatorial e 214 no grupo pernoite, verificou-se que $80,5 \%$ dos pacientes submetidos à colecistectomia ambulatorial receberam alta no dia da cirurgia. Não houve diferença significante em relação à morbidade, prolongamento da internação, taxas de readmissão, qualidade de vida, satisfação do paciente e retorno à atividade normal e ao trabalho. Não foi relatado nenhum caso de mortalidade e as complicações graves foram raras. Todas as lesões da via biliar principal no grupo colecistectomia ambulatorial foram diagnosticadas durante o procedimento. Um caso de pancreatite aguda e outro de insuficiência respiratória necessitando de intubação ocorreram no grupo pernoite. A proporção dos pacientes reavaliados pelo médico, mas não readmitidos, foi semelhante entre os grupos. Desta forma, concluiu-se que a colecistectomia laparoscópica em regime ambulatorial é segura e pode ser realizada com sucesso na maioria dos pacientes. ${ }^{29}$

O HCFMRP-USP, com a finalidade de reduzir a lista de espera para várias operações de media complexidade, no ano de 1996, introduziu o programa dos Mutirões da Saúde. As operações passaram a ser realizadas nos fins de semana, evitando-se assim a ocupação de leitos e horários cirúrgicos destinados ao tratamento dos casos mais complexos.

Entre os anos de 1996 e 2000 foram programados 4 mutirões para colecistectomia videolaparoscópica com a meta de operar 40 pacientes em cada um e consequentemente reduzir a fila de espera para a cirurgia (Figura 2).

Dentre os 160 pacientes selecionados, 159 foram operados e 158 foram avaliados. As comorbidades e seus impactos, avaliados pela classificação da ASA foram mais frequientes no grupo A (Tabela III).

PACIENTES EM FILA DE ESPERA $(n=316)$

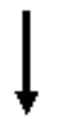

PACIENTES SELECIONADOS $(\mathrm{n}=160)$

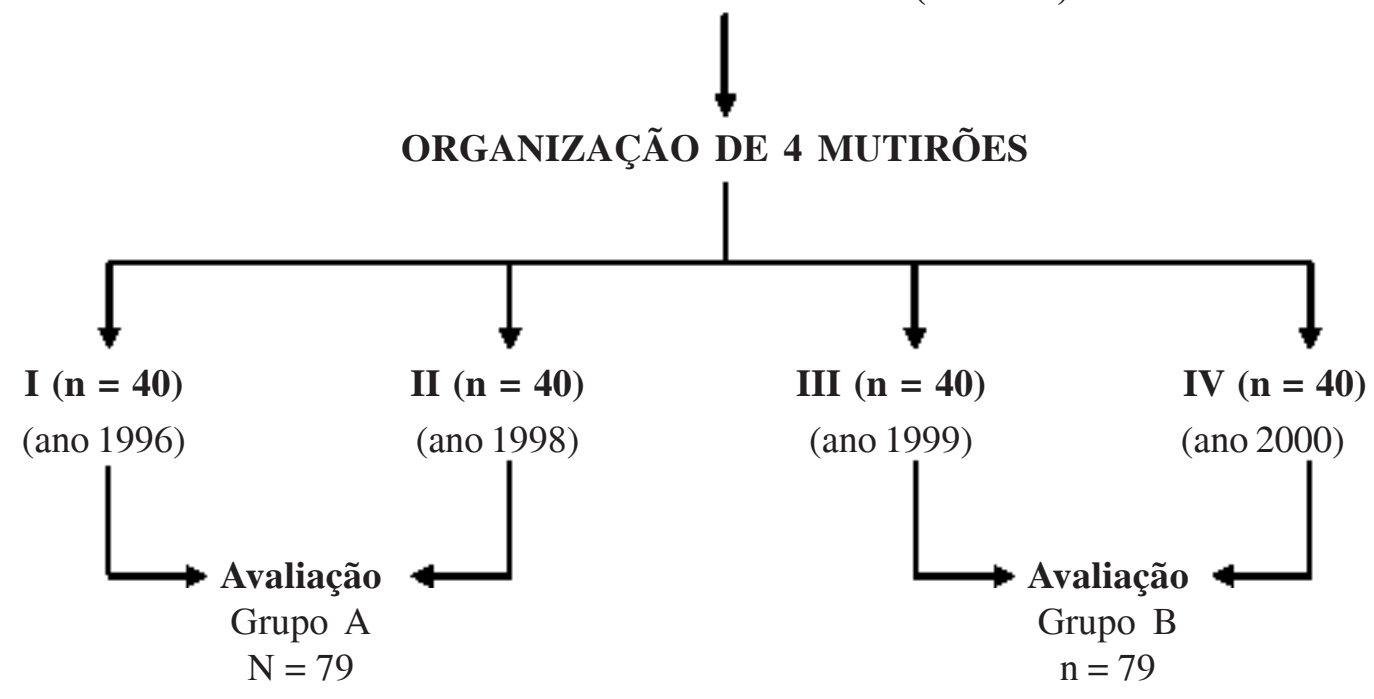

Figura 2: Delineamento do processo de avaliação dos mutirões de colecistectomia videolaparoscópica. 
Tabela III: Caracterização geral dos pacientes

\begin{tabular}{lrrr}
\hline & Grupo A $(\mathrm{n}=79)$ & Grupo B $(\mathrm{n}=79)$ & \multicolumn{1}{c}{$\mathrm{P}$} \\
\hline Idade & $46,92(17-82)$ & $42,80(19-79)$ & 0,007 \\
ASA I & $31(39,2 \%)$ & $48(60,7 \%)$ & 0,005 \\
ASA II + ASA III & $48(60,85 \%)$ & $31(39,3 \%)$ & 0,007 \\
\hline
\end{tabular}

sentavam alterações macroscópicas da vesícula (colecistite aguda e escleroatrófica) enquanto no grupo B estas alterações foram observadas em apenas 2 pacientes $(2,6 \%)$.

A profilaxia da dor com analgésicos e antiinflamatórios não esteroidais e dos vômitos com ondansetrona foi realizada, respectiva-

A avaliação do perfil dos pacientes operados no início da experiência (Grupo A) demonstra que houve flexibilização excessiva dos critérios clínicos e psicosociais para a inclusão no tratamento. A maior frequiência de pacientes classificados como ASA II e III no grupo A justifica a maior incidência de complicações cardio-respiratórias no pós-operatório imediato. Estas ocorrências associadas à falta de vivência com as práticas da cirurgia ambulatorial contribuíram para o prolongamento da permanência hospitalar e para a maior incidência de pernoite neste grupo (Tabelas IV e V).

A duração média das operações no grupo $A$ foi de 90 minutos (25-240) maior do que no grupo B 68,2 minutos (20-180) $(\mathrm{p}=0,002)$. A incidência de perfuração da vesícula foi semelhante nos dois grupos; ocorreu em 14 pacientes do grupo $\mathrm{A}(17,7 \%)$ e em 9 do grupo B (11.4\%). No grupo A, 10 pacientes $(12,7 \%)$ apre-

Tabela IV: Avaliação dos sintomas pós-operatórios.

\begin{tabular}{lrrr}
\hline & Grupo A & Grupo B & P \\
\hline Dor incisional & $48(60,7 \%)$ & $49(62 \%)$ & 0,500 \\
Dor abdominal & $34(43 \%)$ & $18(22,8 \%)$ & 0,005 \\
Vômitos & $18(22,8 \%)$ & $14(17,7 \%)$ & 0,280 \\
Náuseas & $14(17,7 \%)$ & $16(20,2 \%)$ & 0,420 \\
Sintomas Cardiorespiratórios & $10(12,6 \%)$ & $03(3,8 \%)$ & 0,040 \\
Sintomas Neurológicos & $05(6,3 \%)$ & $05(6,3 \%)$ & 0,630 \\
\hline
\end{tabular}

Tabela V: Avaliação da permanência Hospitalar.

\begin{tabular}{lrrr}
\hline & Grupo A & Grupo B & P \\
\hline Pernoite & $45(56,7 \%)$ & $05(6,3 \%)$ & 0,000 \\
$\begin{array}{l}\text { Permanência hospitalar } \\
\text { (horas) }\end{array}$ & $18,37(8,25-26)$ & $7,8(4-24)$ & 0,000 \\
Internações & $05(6,3 \%)$ & $02(2,55 \%)$ & 0,220 \\
\hline
\end{tabular}

mente, em apenas $13(16,4 \%)$ e $2(2,5 \%)$ dos pacientes do grupo A. No grupo B, 63 pacientes $(79,7 \%)$ receberam analgésicos e anti-inflamatórios não esteroidais e 73 (92,5\%) ondansetrona de forma profilática. No grupo A, 53 pacientes $(67,2 \%)$ necessitaram de analgesia e $27(34,2 \%)$ de antieméticos por demanda, enquanto que no grupo B $16(20,3 \%)$ necessitaram analgesia e 4 (5\%) anti-eméticos.

Durante a primeira semana de pós-operatório, 8 pacientes do grupo $\mathrm{A}(10,2 \%)$ e $2(2,6 \%)$ do grupo $\mathrm{B}$ necessitaram de reavaliação em unidades ambulatoriais ou no próprio Hospital. Houve necessidade de 3 readmissões no grupo A; 2 pacientes com vesícula escleroatrófica apresentaram coleperitôneo, um foi tratado mediante drenagem endoscópica e antibioticoterapia (Figura 3) e o outro foi reoperado. O outro paciente, com colecistite aguda, perfuração da vesícula e sangramento moderado do leito hepático durante a colecistectomia, apresentou infecção no sítio cirúrgico. As medidas clínicas e a antibioticoterapia foram suficientes.

A infecção ocorreu em cinco casos $(6,3 \%)$ do grupo A. No grupo B não houve infecção. Não ocorreram óbitos nos dois grupos. A presença de pacientes mais idosos com maior risco cirúrgico no grupo A, associada à duração mais prolongada das operações e o achado de um número maior de vesículas com alterações macroscópicas (inflamação aguda e escleroatrofia) podem justificar a maior incidência de infecção cirúrgica registrada neste grupo.

O programa dos mutirões, iniciado no HCFMRP-USP, foi adotado e aplicado pelo Ministério da Saúde, em âmbito nacional, como estratégia assistencial para o tratamento 


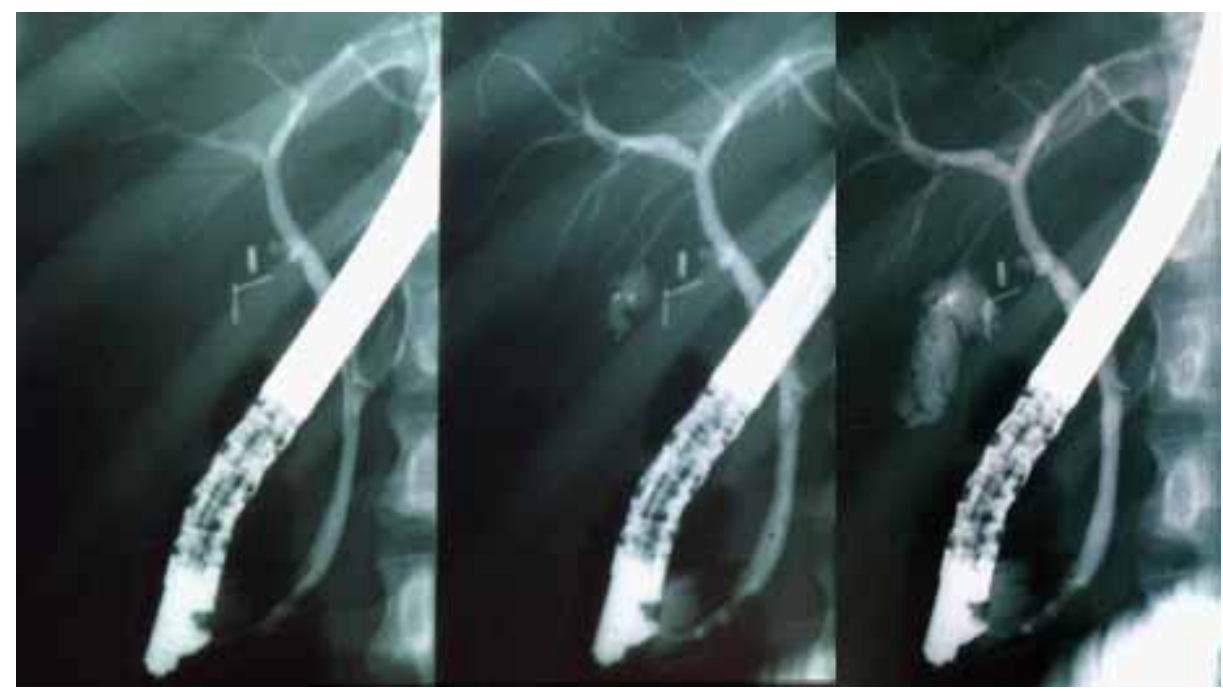

Figura 3: Colangiografia endocópica retrógrada pós colecistectomia videolaparóscópica demonstrando extravasamento do contraste por meio de um pequeno ducto biliar que ficou aberto durante a cirurgia: Fístula biliar - coleperitôneo após colecistectomia de vesícula escleroatrófica.

cirúrgico de várias afecções de média complexidade, a maioria passiveis de resolução em regime ambulatorial. Todavia, esta alternativa paliativa não foi convertida em política pública capaz de equacionar a listas de espera para tratamento cirúrgico de várias afecções de média complexidade.

No ano de 2000, o HCFMRP-USP, a partir do quarto mutirão, disponibilizou dois novos turnos cirúrgicos para a realização rotineira de colecistectomia videolaparoscópica em regime ambulatorial. Entre os anos de 2000 e 2003, foram realizadas 408 colecistectomias em regime ambulatorial. Dentre os pacientes tratados 398 (96,83\%) foram classificados como ASA I e II, a média de permanência global no Hospital foi de 10,19 horas. O pernoite e a internação hospitalar foram indicados em $33(8,02 \%)$ dos pacientes. Os registros de dor incisional, dor abdominal, náuseas e vômitos no pós-operatório foram feitos, respectivamente, em 271 (66,42\%), 52 (12,72\%), 100 (24,50\%), e 99 $(24,26 \%)$ dos pacientes, achados semelhantes aos registrados nos dois últimos mutirões.

\section{3- Serviço integrado de atenção secundária de cirurgia ambulatorial}

A sistematização das atividades com a cirurgia ambulatorial de pequeno porte desenvolvidas, no âmbito da atenção básica, junto ao Centro de Saúde Escola $^{10}$ e a experiência acumulada com a cirurgia ambulatorial de grande porte por meio do programa dos mutirões de colecistectomia realizados no HC-FMRP$\mathrm{USP}^{11}$ e depois como prática rotineira e a organização da rede assistenacial ${ }^{26}$, forneceram subsídios para a proposta de estruturação do Serviço Integrado de Atenção Secundária de Cirurgia Ambulatorial.

A organização de um serviço de cirurgia ambulatorial, no âmbito da atenção secundária, atendia, dentre outros, um dos objetivos educacionais da formação médica na FMRP-USP que preconiza o amplo conhecimento, por parte dos graduandos, do SUS. Ademais, estava alinhada com as necessidades assistenciais e gerenciais do HCFMRP-USP de induzir o deslocamento do tratamento de parte das afecções cirúrgicas de média complexidade para os serviços de atenção secundária, liberando assim os seus serviços para a realização de procedimentos que demandam maior densidade tecnológica.

Desta forma, mediante modelo de co-gestão entre o HCFMRP-USP, a Escola de Enfermagem da USP, o Hospital Beneficência Portuguesa, a Secretaria Estadual de Saúde e a Secretaria Municipal de Saúde foi organizada uma Unidade de Cirurgia Ambulatorial do tipo III no Hospital Beneficência Portuguesa com base em resolução da Secretaria de Estado da Saúde ${ }^{14}$.

Os pacientes cujas afecções eram passíveis de tratamento em regime ambulatorial passaram a ser encaminhados, por meio de referência, das Unidades Básicas e dos Centros de Saúde do município de Ri- 
beirão Preto para o Hospital Beneficência Portuguesa (Hospital Filantrópico de média complexidade).

O Sistema Integrado de Cirurgia Ambulatorial contou com as participações das disciplinas de Cirurgia Gastroenterológica, Cirurgia Vascular, Cirurgia de Cabeça e Pescoço, e Cirurgia Pediátrica. As características dos pacientes, as doenças tratadas, o tempo de permanência do paciente na Unidade de Cirurgia Ambulatorial, e as intercorrências peri-operatórias estão relacionadas nas Tabelas VI e VII.

A média de permanência dos pacientes no serviço, as taxas de pernoite e de infecção estão dentro do esperado. Uma criança apresentou parada cardíaca durante adenoamigdalectomia decorrente de complicação anestésica e faleceu tempos depois constituindo no único óbito de toda a experiência. As taxas de suspensão de cirurgia foram elevadas, em função de problemas relacionados ao transporte social, intercorrências clínicas e ocupação da sala cirúrgica do
Hospital com as operações solicitadas em regime de urgência.

As dificuldades operacionais e administrativas destes programas de co-gestão decorrem da falta de apoio dos departamentos acadêmicos, das escolas médicas e de suporte dos gestores de saúde. Apesar dos benefícios didáticos e assistenciais auferidos, este serviço foi interrompido, mas a experiência ofereceu subsídio técnico e político para a implantação do Hospital Estadual de Ribeirão Preto vinculado ao complexo HC-FMRP-USP / Secretaria de Estado da Saúde. Este Hospital foi inaugurado no ano de 2008 e na área de cirurgia está voltado para a realização de procedimentos de média complexidade, sobretudo em regime ambulatorial.Estas experiências reforçam a necessidade de uma política de saúde e de educação que fortaleça a implantação e a implementação de serviços com os fundamentos conceituais, gerenciais e assistenciais da cirurgia ambulatorial.

Tabela VI: Operações realizadas por especialidade, tempo médio de permanência e taxa de suspensão de cirurgia.

\begin{tabular}{|c|c|c|c|}
\hline & $\mathrm{N}^{\mathrm{o}}$ & Tempo Médio & Suspensão \\
\hline Especialidade cirúrgica & Operações & Permanência (h) & $\mathrm{N}^{\mathrm{o}}$ \\
\hline Vascular & 159 & 9,17 & $(9,4)$ \\
\hline Cabeça e Pescoço & 232 & 10,20 & $(2,6)$ \\
\hline Pediátrica & 155 & 10,27 & $(10,0)$ \\
\hline Digestiva & 156 & 10,26 & $(10,9)$ \\
\hline Total & 702 & 10,50 & $(7,5)$ \\
\hline
\end{tabular}

Tabela VII: Ocorrências por especialidade.

\begin{tabular}{lcccccc}
\hline & \multicolumn{5}{c}{ Especialidades Cirúrgicas } & \\
Ocorrências & Vascular & Cabeça e Pescoço & Pediátrica & Digestiva & No / Total & $(\%)$ \\
\hline Deiscência de Pele & $3 / 159$ & - & - & $11 / 156$ & $14 / 702$ & $(1.9 \%)$ \\
Infecção da Ferida & - & - & - & $4 / 156$ & $4 / 702$ & $(0,6 \%)$ \\
Pernoite & - & $2 / 232$ & $4 / 155$ & $2 / 156$ & $8 / 702$ & $(1,1 \%)$ \\
Óbito & - & $1 / 232$ & - & - & $1 / 702$ & $(0,1 \%)$ \\
\hline
\end{tabular}


Santos JS, Sankarankutty AK, Salgado Jr W, Kemp R, Leonel EP, Castro e Silva Jr O. Outpatient surgery: the concept, organization of the program and its' results. Medicina (Ribeirão Preto) 2008; 41 (3): 274-86.

ABSTRACT: The progress of videoendoscopic surgery, the introduction of short-acting anesthetic drugs with fewer side-effects, as well as the need to teach and provide healthcare, in a scenario which mandates the rational use of available hospital beds has contributed to stretching the limits of outpatient surgery. This is a report on the fundamental conceptual aspects of outpatient surgery, including the technical, ethical and legal issues. The process for the implantation of minor and major outpatient surgical services along with its results are presented. And finally, the technical and political aspects, according to the principles of the Brazilian Unified Health System, of consolidating a network for outpatient surgery involving the Centro de Saúde Escola (Primary), Hospital Estadual (Secondary) and the Hospital das Clínicas (Tertiary), with the cooperation of the Faculdade de Medicina de Ribeirão Preto da Universidade de São Paulo, is discussed.

Keywords: Outpatient Surgery. Ambulatory Surgery. Videolaparoscopy. Education, Medical. Cholecystectomy. Unified Health System.

\section{REFERÊNCIAS BIBLIOGRÁFICAS}

1 - Palumbo LT, Paul RE, Emery FB. Results of primary inguinal hernioplasty. Arch. surg. 1952; 64: 384-94.

2 - Nicholl JH. The surgery of infancy. Br. med. j. 1909; ii:753-6.

3 - Glassow F. Inguinal hernia repair using local anesthesia. Ann. royal coll. surg. engl. 1984; 66: 382-7.

4 - Davis JE, Sugioka K. Seleção do paciente para a grande cirurgia ambulatorial. Avaliação cirúrgica e anestesiológica. Clin. cir. Am. Norte 1987; 67(4): 743-54.

5 - Cohen D, Dillon JB. Anesthesia for outpatient surgery. JAMA 1966; 196(13): 1114-17.

6 - Capella MR, Goldberg P, Quaresma E, Ruiz JS, Kander SL, Araujo EJ. Tratamento cirúrgico ambulatorial da hérnia inguinal na criança: estudo comparativo. J. pediatr. 1982; 52(4): 216-22.

7 - Santos HO, Lino JEO, Lara CS, Cruz MM. Contribuições ao emprego da cirurgia ambulatorial em pediatria. J. pediat. 1984; 57(2): 140-4.

8 - Fonseca FP, Savassi PR. Cirurgia ambulatorial. 2.ed. Rio de Janeiro: Guanabara Koogan, 1999. 536 p.

9 - Peres MAO. Implantação da cirurgia ambulatorial simplificada em hospital comunitário. Análise de resultados. Campinas, 1992. Tese de Mestrado da Faculdade de Ciências Médicas da Universidade Estadual de Campinas.

10 - Santos JS, Sankarankutty AK, Mente ED, Santos AP, Souza Silva C. Cirurgia ambulatorial em assistência primária e saúde da família: avaliação da organização de programa didáticoassistencial para o ensino de graduação. Rev. col. bras. cir. 1999; 26 (Supl 13): 11-13.

11 - Santos JS, Silva MB, Zampar AG, Sankarankutty AK, Campos AD, Ceneviva R. Mutirões de colecistectomia por videolaparoscopia em regime de cirurgia ambulatorial. Acta. cir. bras. 2001; 16(Supl I): 52-6.
12 - Pregler JL, Kapur AP. The development of ambulatory anesthesia and future challenges. Anesthesiol. clin. North America. 2003; 21(2): 207-28.

13 - Cahill H, Jackson I. Day surgery principles and nursing practice. London Balliére Findal, 1997. 238 p.

14 - Resolução da Secretaria da Saúde de São Paulo SS no 02, de 06 de janeiro de 2006, publicado D.O. E.; Poder Executivo, São Paulo, seção 1; 116(5), 7 de janeiro de 2006.

15 - Resolução do CFM, 1802/2006, publicada no D.O.U.; Poder Executivo, Brasília, DF, Seção 1, p. 102, 01 de novembro de 2006.

16 - Resolução do CFM, 1409/1994, publicada no D.O.U.; Poder Executivo, Brasília, DF, Seção 1, p. 8.548, 14 de junho de 1994.

17 - Pasternak L. Preoperative laboratory testing: general issues and considerations. Anesthesiol. clin. North America. 2004; 22(1): 229-42.

18 - Fischer SP. Development and effectiveness of an anesthesia preoperative evaluation clinic in a teaching hospital. Anesthesiology. 1996; 85(1):196-206.

19 - Detmer DE, Buchanan-Davidson DJ. Simpósio sobre sistema de assistência cirúrgica: cirurgia ambulatorial. Clin. cir. Am. Norte. 1982; 62(4): 755-76.

20 - Ferraz EM, Ferraz AAB. Infecção em Cirurgia e profilaxia e tratamento. In: Ferraz AAB, Albuquerque Lima, FEB, Costa Lima EM, Lima MA, Bacelar TS, Andrade Aguiar JL, Ferreira Filho HA, Ferraz EM. Bases da Técnica Cirúrgica e Anestesia, Editora Universitária-UFPE, Pernambuco, 2001 p. 283318.

21 - Mejdahl S, Gyrtrup HJ, Kvist E. Outpatient operation of inguinal hernia in children. Br. j. surg. 1989; 76(4): 406-7.

22 - Kehlet H, Wilmore DW. Multimodal strategies to improve surgical outcome. Am. j. surg. 2002; 183(6): 630-41. 
23 - Geerts WH, Bergqvist D, Pineo G F,. Heit J A,. Samama C M., Lassen MR, Colwell CW. Prevention of venous thromboembolism: American College of Chest Physicians EvidenceBased Clinical Practice Guidelines (8th Edition). Chest. 2008; 133 (6): 381S-453S.

24 - Aguilar-Nascimento JE, Göelzer J. Alimentação precoce após anastomoses intestinais: riscos ou benefícios? Rev. assoc. med. bras. 2002; 48(4): 42-8.

25 - Gan TJ, Meyer T, Apfel CC, Chung F, Davis PJ, Eubanks S, Kovac A, Philip BK, Sessler DI, Temo J, Tramèr MR, Watcha $M$. Consensus guidelines for managing postoperative nausea and vomiting. Anesth. analg. 2003; 97: 62-71.

26 - Santos JS, Scarpelini S, Brasileiro SLL, Ferraz CA, Dallora MELV, Sá MFS. Avaliação do modelo de organização da unidade de emergência do HCFMRP-USP, adotando, como referência, as políticas nacionais de atenção às urgências e humanização. Medicina (Ribeirão Preto). 2003; 36: 498515.
27 - Lopes SLB, Santos JS, Scarpelini S. The implementation of the medical regulation office and mobile emergency attendance system and its impact on the gravity profile of nontraumatic afflictions treated in a university hospital: a research study. BMC health serv. res. 2007; 7: 173.

28 - Ceneviva R, Santos JS, Mente ED, Sankarankutty AK, Castro e Silva Jr O. Colecistectomia laparoscópica como cirurgia ambulatorial. Rev. col. bras. cir., Vol XXVI Supl 13: 425,1999 .

29 - Gurusamy K, Junnarkar S, Farouk M, Davidson BR. Metaanalysis of randomized control trials on the safety and effectiveness of day-case laparoscopic cholecystectomy. Br. j. surg. 2008; 95: 161-8.

Recebido para publicação em 20/08/2008

Aprovado para publicação em 30/09/2008 